\author{
Naoya Wada ${ }^{\mathrm{a}}$, Kumiko Takamatsu ${ }^{\mathrm{a}}$, Mahoto Takeda ${ }^{\mathrm{a}}$, Masaki Takeguchi ${ }^{\mathrm{b}}$, \\ Marie-Gevenieve Blanchin ${ }^{\mathrm{c}}$ \\ ${ }^{a}$ Department of Mechanical Engineering and Materials Science (SEISAN), Yokohama National University, Yokohama, Japan \\ ${ }^{\mathrm{b}}$ National Institute for Materials Science, Tsukuba, Japan \\ ${ }^{c}$ Laboratoire de Physique de la Matière Condensée et Nanostructures, Université Claude Bernard Lyon I, Villeurbanne, France
}

\title{
The microstructure and magnetic properties of nano-scale Fe magnetic particles precipitated in a $\mathrm{Cu}-\mathrm{Fe}$ alloy
}

\begin{abstract}
The evolution of nano-scale Fe particles precipitated in a $\mathrm{Cu}-\mathrm{Fe}$ alloy has been examined from the viewpoint of the relationship between the microstructure and magnetic properties, using conventional, high-resolution and Lorentz electron microscopy, and magnetic measurements. It has been revealed that spherical Fe particles have a tendency to align along the $<001>$ direction in a $\mathrm{Cu}$ matrix at the early stage of precipitation. A twin-like structure developed when spherical Fe particles grew to approximately 40 to $60 \mathrm{~nm}$ in size. Lorentz microscopy was successfully applied to determine the direction and magnitude of the magnetic momentum of Fe particles.
\end{abstract}

Keywords: $\mathrm{Cu}-\mathrm{Fe}$ alloy; Lorentz microscopy; Precipitation; Nano-scale particle

\section{Introduction}

Ferromagnetic particles have recently attracted considerable attention, as it may be possible to use them in various magnetic devices and next-generation storage applications. Despite the desirability of reducing the size of magnetic domains to further improve magnetic storage devices, refining the magnetic recording unit does not always lead to improvements, due, for example, to the appearance of superparamagnetism when the particle size is of the order of several nanometers. In addition to the innovative practical applications, the discovery of the giant magnetoresistanceeffect in substances containing nano-granular magnetic particles has made it necessary to conduct fundamental research to understand the structures and the magnetic properties of nano-scale magnetic particles. According to the equilibrium phase diagram, the fcc $\gamma$-Fe phase is stable at temperatures between 1185 and $1667 \mathrm{~K}$, whereas bcc $\alpha$ Fe becomes stable below $1185 \mathrm{~K}$. However, Bitter reported that small $\mathrm{Fe}$ particles had an fcc structure even below $1185 \mathrm{~K}$ [1]. Kinsman claimed that $\alpha$-Fe particles comprised $\{112\}$ twins of the order of 5 to $50 \mathrm{~nm}$ in width [2]. There have been a number of studies on the structure and the magnetic properties of $\mathrm{Cu}-\mathrm{Fe}$ alloys investigated by Mössbauer measurements and X-ray and neutron diffraction. Abraham has reported that neutron diffraction revealed $\mathrm{Fe}$ precipitates in a $\mathrm{Cu}$ matrix to be antiferromag- netic $\gamma$-Fe [3]. Using low-temperature X-ray diffraction, Tsunoda pointed out that the lattice modulation of nanoscale Fe particles can be described as a periodic spin wave [4]. Gonser et al. investigated the Néel temperature of $\gamma$-Fe precipitates in a $\mathrm{Cu}$ matrix by Mössbauer measurements [5]. Mössbauer and X-ray analysis give us locally-averaged information about the structure. The magnetic properties of nano-scale particles are, however, also very sensitive to their local microstructures. Thus it is essential to investigate how the microstructure relates to the magnetic properties. Transmission electron microscopy (TEM) and Lorentz microscopy allow us to observe the details of microstructures and the distribution of the magnetic domains in a localized area. The purpose of this study was to investigate the relationship betwe $\mathrm{e}^{\wedge}$ en the precipitation behavior of nano scale $\mathrm{Fe}$ particles and the magnetic properties in a $\mathrm{Cu}-\mathrm{Fe}$ alloy.

\section{Experimental procedure}

The present study used $\mathrm{Cu}-3$ at.\% Fe alloy specimens. The alloy ingots were produced by arc-melting in an argon atmosphere. After subsequent annealing and rolling, diskshaped specimens of thickness $200 \mu \mathrm{m}$ were obtained and sealed in an evacuated silica tube. The specimens were solution-treated at $1323 \mathrm{~K}\left(1050^{\circ} \mathrm{C}\right)$ for $180 \mathrm{~min}$, then quenched in iced water at $273 \mathrm{~K}\left(0^{\circ} \mathrm{C}\right)$. After the solution treatment, isothermal annealing was conducted at $873 \mathrm{~K}$ $\left(600^{\circ} \mathrm{C}\right)$ for various periods. The TEM specimens were finally obtained by electro-polishing. TEM observations were performed using a $200 \mathrm{kV}$ HITACHI H- 800 and a JEOL JEM-3010F transmission electron microscopes. The magnetic domain structure was observed in the low-angle diffraction (LAD) mode using a TOPCON EM-002BL Lorentz microscope. SQUID measurements were conducted to obtain the magnetization vs. temperature curves from $5 \mathrm{~K}$ to $350 \mathrm{~K}$ under a small applied-field of $7958 \mathrm{~A} \mathrm{~m}^{-1}$ (100 Oe) using a Quantum Design MPMS-XL SQUID Magnetometer.

\section{Results and discussion}

\subsection{Precipitation processes in a $\mathrm{Cu}-\mathrm{Fe}$ alloy}

TEM observations were conducted to examine the precipitation processes occurring in a $\mathrm{Cu}-\mathrm{Fe}$ alloy. Figure 1 


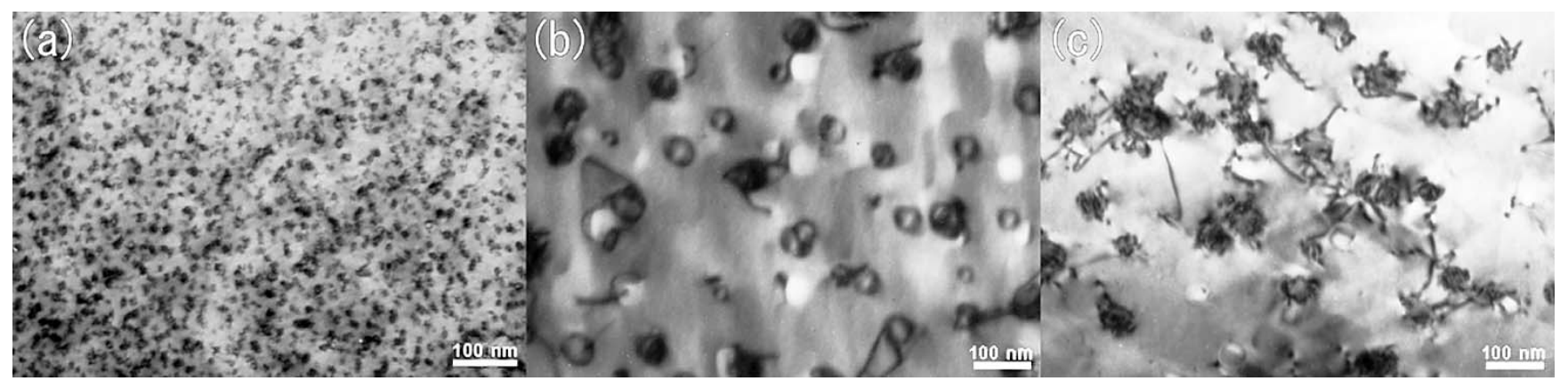

Fig. 1. Bright field TEM images of Cu-3 at.\% Fe alloy aged at $873 \mathrm{~K}$ for (a) $500 \mathrm{~min}$, (b) $2000 \mathrm{~min}$, and (c) $20000 \mathrm{~min}$.

shows bright-field TEM images of $\mathrm{Cu}-3$ at.\% $\mathrm{Fe}$ alloy annealed isothermally at $873 \mathrm{~K}$ for (a) 500 minutes, (b) $2000 \mathrm{~min}$, and (c) $20000 \mathrm{~min}$. No precipitates were found immediately after iced-water quenching, indicating that Fe atoms are still in supersaturated solid solution. After annealing for $100 \mathrm{~min}$, very small precipitates appeared in the $\mathrm{Cu}$ matrix. After further annealing at $873 \mathrm{~K}, \mathrm{Fe}$ precipitates evolved as spherically-shaped particles 10 to $20 \mathrm{~nm}$ in size. The Fe precipitates continued growing to $40 \mathrm{~nm}$ in size, and finally incoherent precipitates over $40 \mathrm{~nm}$ in size appeared, accompanied by interfacial dislocations. Figure $2 \mathrm{a}$ shows the microstructure of the specimen annealed for $1000 \mathrm{~min}$, corresponding to the late coherent stage of the precipitation process. An interesting feature in the formation of the precipitates may be seen in this micrograph, namely that pairs of precipitates of similar size were linearly arranged along the $<001>$ direction of the $\mathrm{Cu}$ matrix. This tendency was also found in a $\mathrm{Cu}-\mathrm{Co}$ alloy, reported by Takeda et al. [6]. In $\mathrm{Cu}-\mathrm{Fe}$ the tendency is less marked than in a $\mathrm{Cu}-\mathrm{Co}$ alloy. After the precipitates grew to 35 to
$45 \mathrm{~nm}$ in size, narrow stripes approximately 5 to $10 \mathrm{~nm}$ wide appeared in the Fe particles, as shown in Fig. 2b. The average width of the layered domains comprising the stripes increased as the particles became larger.

\subsection{High resolution TEM observations}

Previous studies on $\mathrm{Cu}-\mathrm{Fe}$ alloys have adopted the interpretation that both fcc $\gamma$-Fe and bcc $\alpha$-Fe are present in the Fe precipitation process. However, the details of the transition between the two phases have not been fully investigated. We conducted HRTEM observations, SQUID magnetic property measurements, and Lorentz microscopy to investigate this point. Figure 3 shows high-resolution TEM images of the coherent (a), and incoherent precipitates (b), respectively. Lattice fringes were observed outside the Fe particles, corresponding to the (200) planes of the $\mathrm{Cu}$ matrix. Two lattice fringes perpendicularly crossing with each other are observed in a Fe particle in Fig. 3a. Using the (200) lattice spacing value of the $\mathrm{Cu}$ matrix as a reference,
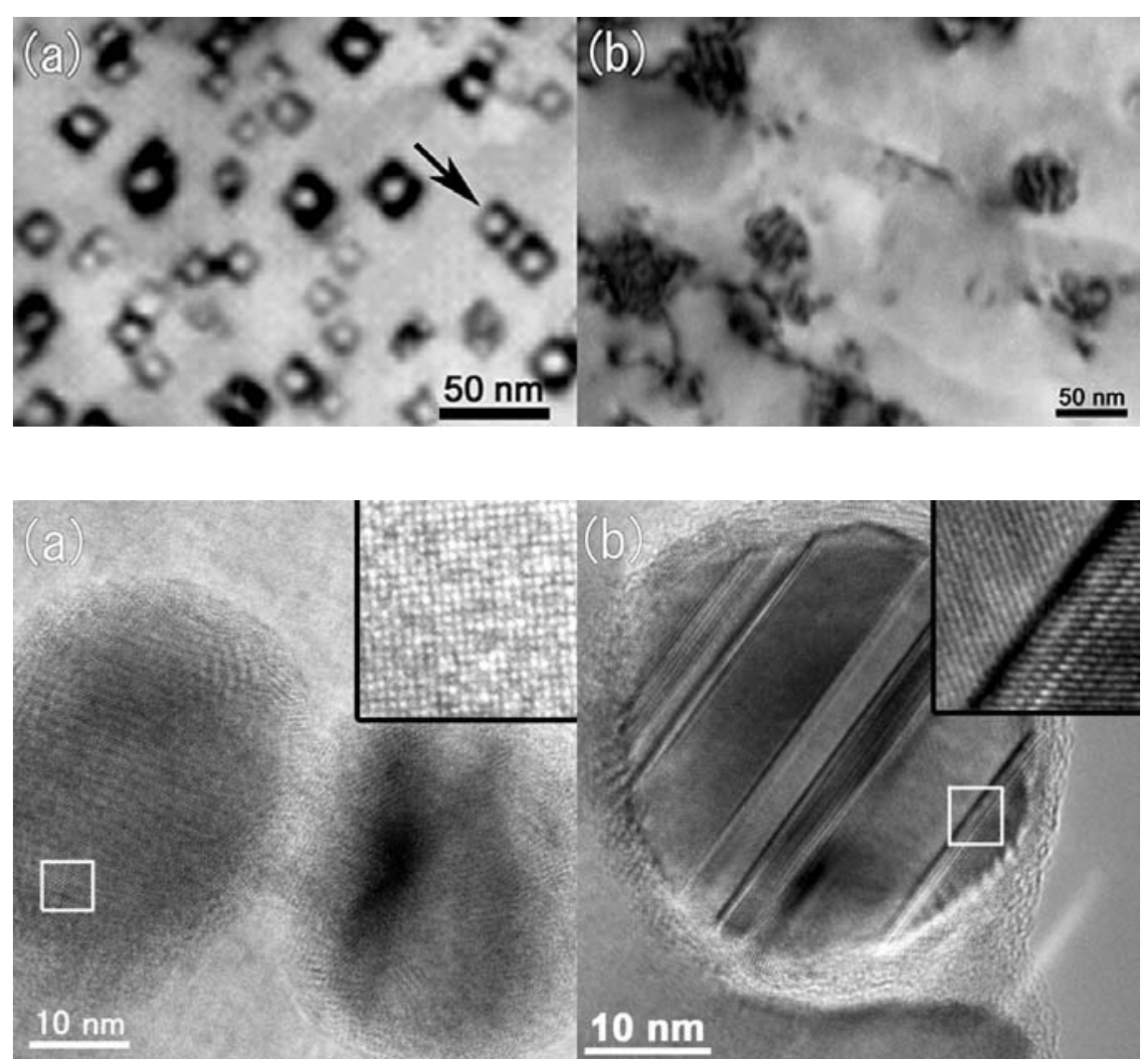

Fig. 2. Bright field TEM images of $\mathrm{Cu}-3$ at. $\%$ Fe alloy aged at $873 \mathrm{~K}$ (a) The arrow indicates a pair of linearly arranged Fe particles, (b) twin-like structure.

pitates in a $\mathrm{Cu}-3$ at. $\%$ Fe alloy aged at $873 \mathrm{~K}$
peci(a) linearly arranged Fe particles, (b) twin. Inset top-right is an enlarged image of the microstructure of the particle. 
(a)

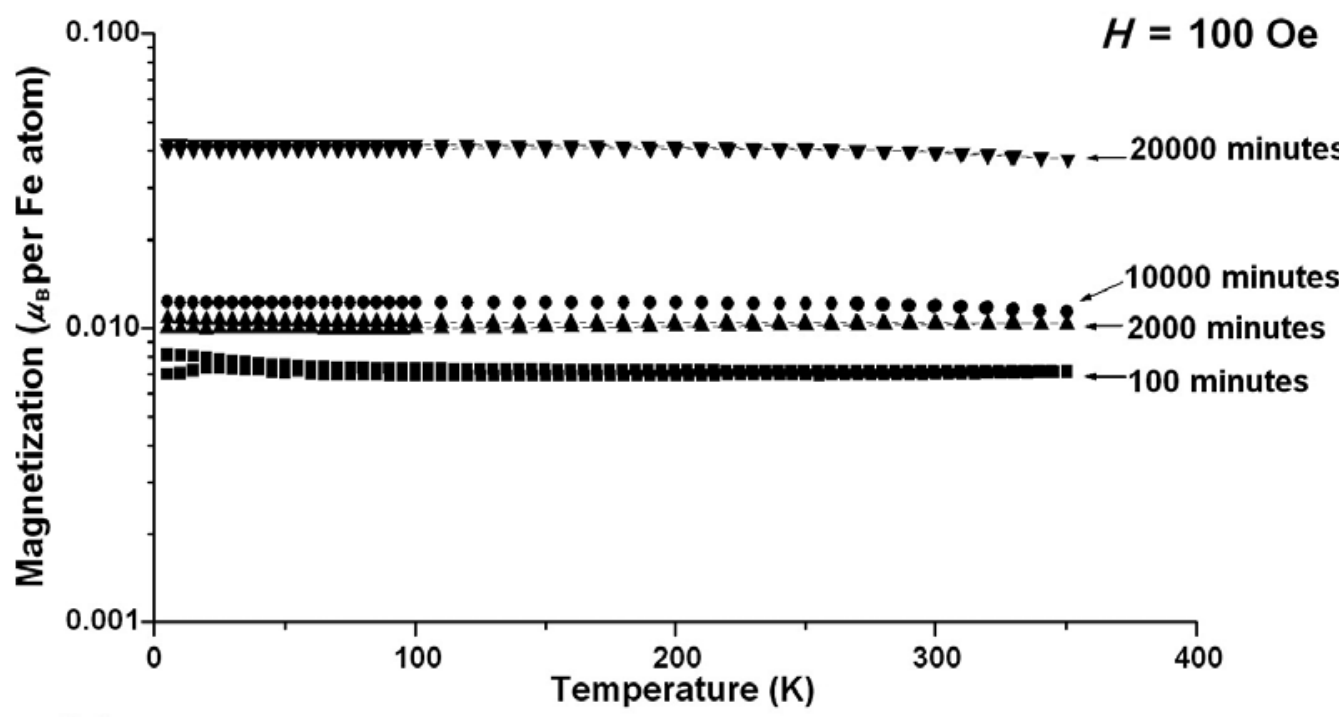

Fig. 4. (a) Magnetization vs. temperature curves for $\mathrm{Cu}$ 3 at.\% Fe alloy aged at $873 \mathrm{~K}$ for $100 \mathrm{~min}, 2000 \mathrm{~min}$, $10000 \mathrm{~min}$, and $20000 \mathrm{~min}$. (b) ZFC curve obtained from the specimen annealed for 2000 min (c) FC curve obtained from the specimen annealed for $2000 \mathrm{~min}$. Vertical scale is Bohr magneton per

(b)

(c)
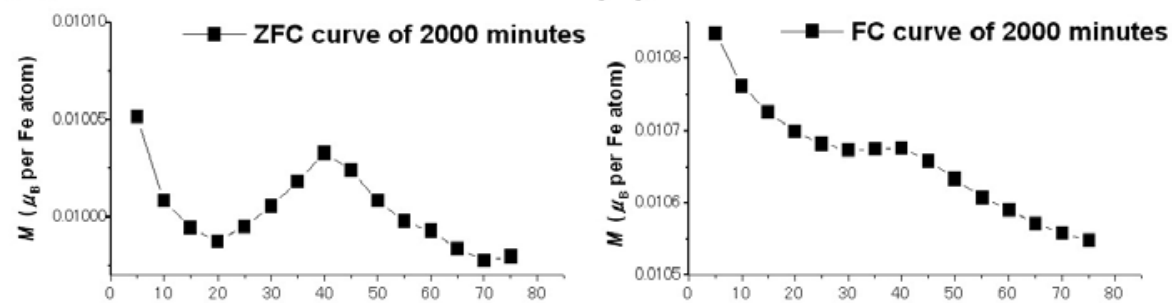

the widths of the two orthogonal lattice fringes are estimated at $0.177 \mathrm{~nm}$ and $0.180 \mathrm{~nm}$, respectively. We calculated the lattice spacings under the assumption that the crystal structure of Fe particles is fcc or bcc. This calculation showed that the lattice fringes observed in the TEM images could be from either the $\{200\}$ planes of the fcc structure or from $\{110\}$ planes of the bcc structure. Thus two possibilities are suggested that coherent $\mathrm{Fe}$ particles in a $\mathrm{Cu}$ matrix have either the fcc structure or the bcc structure. Figure $3 b$ shows a microstructure different from the feature shown in Fig. 3a. The Fe particles consist of layered domains. The width of the lattice fringes inside the layers is approximately $0.203 \mathrm{~nm}$. This value almost corresponds to the lattice spacing of $\{110\}$ planes of $\alpha-\mathrm{Fe}$ in a bcc structure. The lattice fringes are symmetrically inclined at an angle 55 degrees to the interfaces of the layers. It is probable that the lattice fringes observed in Fig. $3 b$ correspond to the $\{110\}$ planes of bcc Fe. This result also agrees with the fact that the twin plane of bcc bulk Fe is the $\{112\}$ plane. This present result is similar to that obtained by Kubo et al. [7].

\subsection{Measurements of magnetic properties}

Magnetization vs. temperature $(M(T))$ measurements were performed using a SQUID magnetometer to investigate the magnetic properties of the $\mathrm{Cu}-3$ at.\% Fe specimens. Figure $4 \mathrm{a}$ shows $M-T$ curves obtained for specimens annealed at $873 \mathrm{~K}$ for $100 \mathrm{~min}, 2000 \mathrm{~min}, 10000 \mathrm{~min}$, and $20000 \mathrm{~min}$, under an applied field of $7958 \mathrm{~A} \mathrm{~m}^{-1}$ $(100 \mathrm{Oe})$ in a temperatures range $5 \mathrm{~K}$ to $350 \mathrm{~K}$. The magnetization of $M(T)$ curve was larger, the longer the annealing time. This indicates that Fe particles tend to become ferro- magnetic with increasing annealing time. Figure $4 \mathrm{~b}$ and $\mathrm{c}$ show the ZFC and FC curves obtained from the specimen annealed for $2000 \mathrm{~min}$. Both curves exhibit a small cusp at around $40 \mathrm{~K}$. Similar features were found in other specimens annealed for $\leq 10000 \mathrm{~min}$, except the specimen annealed for $100 \mathrm{~min}$. These cusps are commonly observed in $M(T)$ curve and associated with antiferromagnetism. Table 1 shows the relationship between aging time and the temperature at which the small cusp appeared. All the values are close to the Néel temperature of $44 \mathrm{~K}$ for bulk Fe [8]. We refer that the cusps originate from the formation of antiferromagnetic $\gamma$-Fe particles. As shown in Fig. 4a, the maximum magnetization value of 0.04 ( $\mu_{\mathrm{B}}$ per Fe atom) of the sample annealed for 20000 min was much higher than that of the others. No cusp was observed at around $40 \mathrm{~K}$ in this sample. These results indicate that $\gamma$-Fe transforms to $\alpha$-Fe after a long period of annealing without plastic deformation. In the TEM observations, there is the possibility that the transition from fcc to bcc is caused by thinning of the foil during electropolishing, as suggested by Kubo [7].

Table 1. Relationship between the average particle size and the temperature at which small cusps appeared on $M(T)$ curves.

\begin{tabular}{|c|c|c|}
\hline $\begin{array}{c}\text { Aging time } \\
(\mathrm{min})\end{array}$ & $\begin{array}{c}\text { Average particle size } \\
(\mathrm{nm})\end{array}$ & $\begin{array}{c}\text { Cusp Temperature } \\
(\mathrm{K})\end{array}$ \\
\hline 500 & 14.14 & 55 \\
1000 & 19.77 & 40 \\
2000 & 39.15 & 40 \\
5000 & 44.24 & 40 \\
10000 & 52.70 & 55 \\
\hline
\end{tabular}


N. Wada et al.: The microstructure and magnetic properties of nano-scale Fe magnetic particles in a $\mathrm{Cu}-\mathrm{Fe}$ alloy
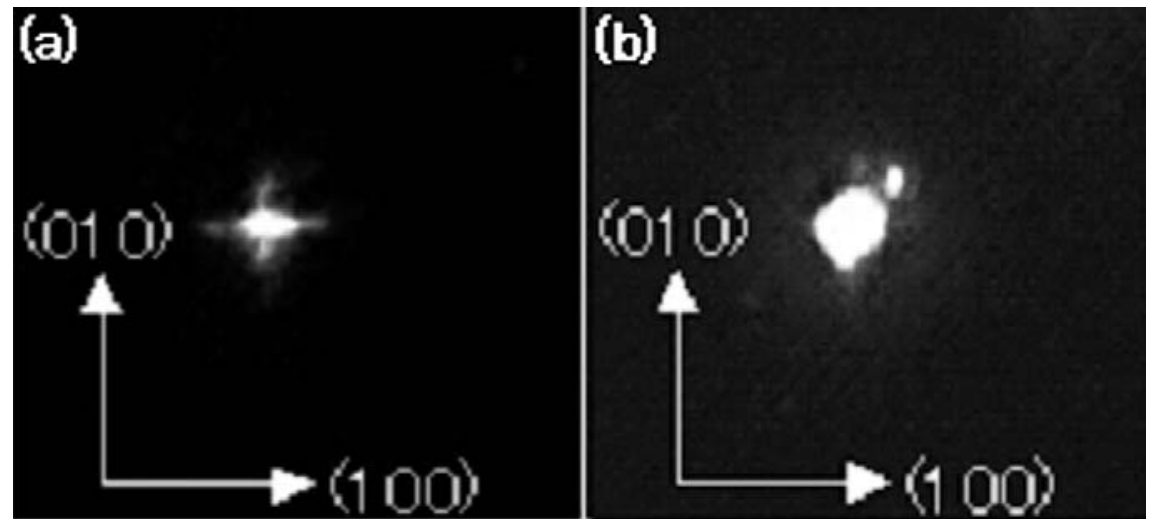

Fig. 5. (a) Electron diffraction spot of (220) reflection obtained from a specimen containing Fe particles $\sim 15 \mathrm{~nm}$ in size using Lorentz microscope. Satellite spots are seen along the [100] and [010] directions. The beam deflection corresponds the direction of magnetization of $\mathrm{Fe}$ particles. (b) Electron diffraction spot obtained from a specimen containing $\mathrm{Fe}$ particles $\sim 50 \mathrm{~nm}$ in size. Satellite spots are observed in the [110] direction.

However, the specimens for SQUID magnetic measurements were not polished or thinned. Therefore, $\gamma$-Fe precipitates are likely to transform spontaneously to $\alpha$-Fe without a change of strain field around the precipitates. If we regard the cusp as the Néel temperature, the present results suggest that the particles retained the gamma structure up to a size of $50 \mathrm{~nm}$ after which they transformed to $\alpha-F e$.

\subsection{Lorentz microscopy observations}

In order to verify the direction and magnitude of the magnetization of Fe particles, we conducted low-angle diffraction (LAD) using Lorentz microscopy [9]. When electrons travel in magnetic materials, the incident electrons are deflected by the Lorentz force due to magnetization of the specimen. The deviation from the normal Bragg reflections due to the Lorentz force can be detected using Lorentz microscopy. We examined eight specimens with particle sizes of approximately 10 to $60 \mathrm{~nm}$. Two examples of LAD are shown in Fig. 5. Previous studies have reported that the easy axis of magnetization of bcc $\alpha$-Fe is almost parallel to the $<100>$ orientation. The deviation of the Bragg reflection occurs in the direction perpendicular to both the electron beam and magnetic field. Thus it is essential to observe the deflection of the electron beam from the [001] zone axis. Satellite spots are seen along the [100] and [010] directions. Because the particles are very small, the spots are rather fuzzy, so the deflection spots assume to shape of a cross with arrows along [100] and [010]. We also observed $<110>$ additional spots in Fig. 5b when particles have a size exceeding $45 \mathrm{~nm}$. As far as the elongation along $<100>$ is concerned, the present result confirms that the easy axis of magnetization of $\alpha-\mathrm{Fe}$ is the $<100>$ orientation. The origin of the $<110>$ additional spots has not yet been identified. Tsunoda et al. reported that a lattice modulation occurred in a small Fe particle [4]. The $<110>$ extra spots may relate to such a lattice modulation. Using LAD, the intensity of magnetization can also be estimated. We measured the distance between the fundamental and extra spots, and estimated the intensity of magnetization at each stage of the precipitation process. The Lorentz force generated by magnetic field $\boldsymbol{B}$ is given by:

$\boldsymbol{F}=-e(\boldsymbol{v} \times \boldsymbol{B})$

where $e$ is the electric charge and $\boldsymbol{v}\left(v_{x}, v_{y}, v_{z}\right)$ is the electron velocity. By Newton's law, the rate of change of the momentum of an electron caused by the Lorentz force is written as:

$$
\left\{\begin{array}{l}
m \frac{\mathrm{d} v_{x}}{\mathrm{~d} t}=e v_{y} B \\
m \frac{\mathrm{d} v_{x}}{\mathrm{~d} t}=-e v_{x} B \\
m \frac{\mathrm{d} v_{z}}{\mathrm{~d} t}=0
\end{array}\right\}
$$

where $m$ is the electron mass. Since the deflection of the electrons is very small, the direction of $\boldsymbol{B}$ is set along the $z$ axis. By setting the initial condition and the velocity of the incident electron to $v$, we obtain the following equation:

$$
\left\{\begin{array}{l}
x=\frac{m v}{e B} \sin \frac{e B}{m} t \\
y=\frac{m v}{e B}\left(\cos \frac{e B}{m} t-1\right)
\end{array}\right\}
$$

As is evident from Eq. (3), the incident electrons rotate with a radius of $\frac{m v}{e B}$. Hence, the deflection angle $\varphi$ is written as

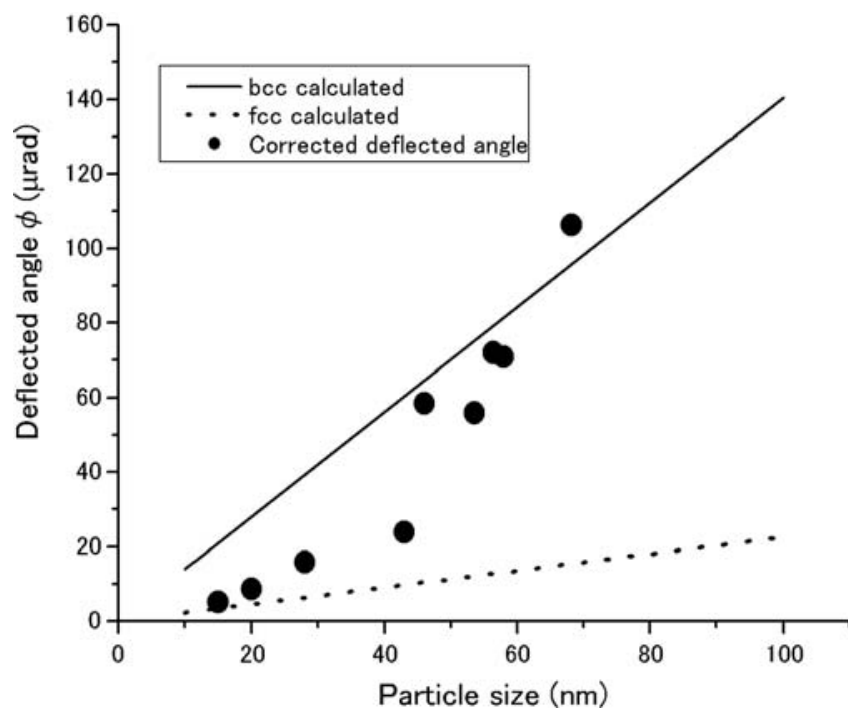

Fig. 6. The relationship between deflection angle and particle size. The solid and broken lines refer to the calculated angles for the $\alpha$-Fe and $\gamma$-Fe particles, respectively. The deflection angles experimentally obtained increases at $\sim 45 \mathrm{~nm}$ in size. The large increment of the angle suggests that the structural transformation from $\gamma$-Fe to $\alpha$-Fe occurs at a particles sizes of around $45 \mathrm{~nm}$. 
follows:

$\varphi=\frac{e B \mathrm{~d} \lambda}{h}$

where $\lambda$ is the wavelength of the electron beam and $h$ is Planck's constant. The results of the present investigation are shown in Fig. 6. The solid and broken lines refer to the calculated angles for the $\alpha$-Fe and $\gamma$-Fe particles, respectively. We found that the experimental values of the deflection angles are somewhat greater than calculated with Eq. (4). An approximate correction for the deflection angle has been made by shifting the value obtained for smallest particle size $(15 \mathrm{~nm})$ onto the fcc calculated line. After this correction, the experimental values obtained from the particles greater than $45 \mathrm{~nm}$ in size tended to deviate from the fcc calculated value and transfer to the bcc theoretical curve. These results seem consistent with structural transformation $\gamma \rightarrow \alpha$, described in Section 3.3. The reason for the difference between the experimental and calculated values is not yet clear. The corrected angles obtained from particles of size less than $45 \mathrm{~nm}$ lie close to the theoretical value of $\gamma$-Fe, while those from particles larger than $45 \mathrm{~nm}$ roughly coincide with the theoretical value of $\alpha$-Fe. This suggests that the structural transformation from $\gamma-\mathrm{Fe}$ to $\alpha$-Fe occurs when the particle grows to around $45 \mathrm{~nm}$ in size. This value is somewhat greater than the value obtained from the magnetic measurements. This difference may be attributed to the size distribution. Thus the present study has obtained some important information about the microstructure and the magnetic properties of nano-scale Fe particles formed in a $\mathrm{Cu}$ matrix. The details of the magnetic domain structures provide a very interesting subject for further study. Lorentz TEM in Foucault mode is suggested as a suitable approach to gain information on the structure. A further study is intended by the present research group.

\section{Conclusions}

We have examined the relationship between the microstructure and the magnetic properties of $\mathrm{Cu}-3$ at.\% Fe using TEM observations, SQUID magnetic measurements, and Lorentz microscopy. It has been found that:

1. Fe particles gradually evolved from spherical coherent particles to incoherent particles.

2. At the late coherent stage, $\alpha$-Fe particles comprise layered domains with a twin relation.

3. The particles with twin-like structure most probably have a bcc structure.

4. The transformation from $\gamma$-Fe to $\alpha$-Fe occurs when $\mathrm{Fe}$ particles grow to around $45 \mathrm{~nm}$ in size.
We would like to acknowledge to Profs. Z. Hiroi of the University of Tokyo and Dr. M. L. Jenkins of the University of Oxford for critical readings and useful comments. This work was supported in part by Grants-in-Aid for Scientific Research from JSPS of Japan (No. 18560643).

\section{References}

[1] F. Bitter, A.R. Kaufmann: Phys. Rev. 56 (1939) 1044. DOI:10.1103/PhysRev.56.1044

[2] K.R. Kinsman, J.W. Sprys, R.J. Asaro: Acta Metall. 23 (1975) 1431. DOI:10.1016/0001-6160(75)90152-2

[3] S.C. Abrahams, L.G. Guttman, J.S. Kasper: Phys. Rev. 127 (1962) 2052. DOI:10.1103/PhysRev.127.2052

[4] Y. Tsunoda: J. Phys. Cond. Matt. 1 (1989) 10427. DOI:10.1088/0953-8984/1/51/015

[5] U. Gonser, C.L. Meechan, A.H. Muir, H. Wiedersich: J. Appl. Phys. 34 (1963) 2373. DOI:10.1063/1.1702749

[6] M. Takeda, H. Yamada, S. Yoshida, K. Fujii, T. Endo, J. Van Landuyt: Phys. Stat. Sol. A 198 (2003) 436. DOI:10.1002/pssa.200306634

[7] H. Kubo, V. Uchimoto, K. Shimizu: Metal Sci. J. 9 (1985) 153.

[8] H.P.J. Wijn: Magnetic Properties of Metals, Springer-Verlag, Ber$\operatorname{lin}(1991)$

[9] J.N. Chapman, M.R. Scheinfein: J. Magn. Magn. Mater. 200 (1999) 729. DOI:10.1016/S0304-8853(99)00317-0

(Received September 3, 2008; accepted August 31, 2009)

\section{Bibliography}

DOI $10.3139 / 146.110288$

Int. J. Mat. Res. (formerly Z. Metallkd.)

101 (2010) 3; page $361-365$

(C) Carl Hanser Verlag GmbH \& Co. KG

ISSN 1862-5282

\section{Correspondence address}

Prof. Mahoto Takeda

Department of Mechanical Engineering and Materials Science

(SEISAN), Yokohama National University

79-5 Tokiwadai Hodogaya, Yokohama 240-8501, Japan

Tel.: +81453393848

Fax: +81453393855

E-mail: t1k4d1@ynu.ac.jp

You will find the article and additional material by entering the document number MK110288 on our website at www.ijmr.de 